\title{
Product Reviews in Travel Decision Making
}

\author{
Francesco Ricci ${ }^{\text {a }}$, and \\ René T.A. Wietsma ${ }^{b}$ \\ a e-Commerce and Tourism Research Laboratory \\ ITC-irst, Trento, Italy \\ ricci@itc.it \\ ${ }^{\mathrm{b}}$ Human Media Interaction \\ University of Twente, \\ Enschede, The Netherlands \\ rta@wietsma.nl
}

\begin{abstract}
Product reviews or user opinions represent a peculiar information source that can be exploited in supporting travel decision making. Product reviews provide other user's experiences and, when consulted during a decision making process, tend to increase the user's confidence in the decision by providing a feeling of greater objectivity in the product description compared with commercial information. Notwithstanding their value, until now, product reviews have not been integrated in travel recommender systems, they have been used only in dedicated web sites. In this paper we present the results of a first step towards the introduction of product reviews in a travel recommender system, namely the outcome of a user behaviour survey. In this survey, we investigated the possible roles/functions of product reviews in the product selection decision process. In this paper we show that there is a significant difference in the role of reviews with respect to the product involved in the decision process (e.g. hotel vs. activity) and we demonstrate that advanced users, i.e., users who are familiar with online booking platforms and with consumer-opinion platforms, are more interested in consulting reviews that criticize the product and express a negative evaluation of it.
\end{abstract}

Keywords: travel recommender systems; consumer behaviour; empirical evaluation.

\section{Introduction}

A recent article in the Wall Street Journal points out the success factors of travel blogs, websites where people can post their travel experience in the form of a review (Saranow, 2004). This article indicates that people look for different ways to obtain and share more objective pieces of information about tourist products. Where a 
popular travel booker system may say: "This is great" or "Try this out", the personal reviews are expected to provide a more nuanced view, supported by ratings and rich media data (photos, video, etc.)

In fact, that article points out the importance of consumer generated media (CGM), a definition with encompasses the millions of consumer-generated comments, opinions and personal experiences posted in publicly available online sources on a wide range of issues, topics, products and brands. Experts have estimated that nowadays, one can find more than 1.4 billion CGM comments and this amount is increasing annually with 30\% (Blackshaw, 2005).

CGM could be highly beneficial in the tourist context since one of the major problems of the travel domain is the lack of a direct experience of tourist products (Senecal \& Nantal, 2004). Products, or better said tourist services, lacks the feature of "trybefore-buy" or "return in case the quality is below expectance" (Buhalis, 2003). This implies that (online) tourist product buying involves a certain amount of risk taking, which could be lowered by providing the user a better and more reliable product description. Research has already proved that consumers put more trust in fellow consumers than in content provided by (marketing) agencies (Blackshaw \& Nazzaro, 2005). Another important aspect of the travel domain, is that a user buys more an overall experience rather than a product (Hennig-Thurau et al., 2004; Senecal \& Nantal, 2004). This experience involves three phases: anticipation (before), consumption (during) and memory (after). Reviews of fellow travellers could contribute to the first and last phase by providing a way to gather information of similar travellers or to process your own travel experiences.

In this paper, we focus on one type of CGM: user generated product reviews (PRs). Product reviews can be described as a subjective piece of non-structured text describing the user's product knowledge, experiences and opinions, together with a final product rating. On the web, many products advise guides can be found which offer basic functionalities to find products, their specification and the user reviews (BBC, 2004). These sites can be classified, as consumer-opinion platforms which incorporate 'word-of-mouth' principles, i.e.: facilitating the exchange of product opinions between non-experts (Hennig-Thurau et al., 2004; Jin et al., 2002).

In the travel domain, Tripadvisor (Tripadvisor.com, 2005) is an example of a popular consumer-opinion platform. On this website, one can find information and opinions about tourist products (hotels, activities) grouped by geographic location and product type. These products are enriched with product reviews, written by non-experts. In Tripadvisor a product review has a title which represents the general opinion, a rating 
(1-5) and (review) content which can be regarded as the motivation for the given rating. The average product rating, the number of ratings and three summarized user opinions are shown to the user when he browses the product listed on this website. Tripadvisor has more than 2.6 million product reviews and is visited by more than 13 million unique visitors per mouth (PR Newswire, 2005).

Our research focuses on improving the user acceptance and the explanation functionality of product recommender systems by augmenting recommendations with relevant product reviews. Recommender systems are applications exploited on eCommerce sites to suggest products and provide consumers information to facilitate the decision process (Schafer et al., 2001) (Ricci, 2002). These systems offer a personalized set of recommendations by incorporating the user wishes in a user model and exploiting appropriate recommendation algorithms to map the user model into targeted product suggestions.

In this paper we will describe the results of a user behaviour study that involved 29 users. The goal of this user behaviour study was to investigate the potential roles of product reviews in the decision process and to derive design principles for an augmented travel recommender system. The study consisted of two parts: a user behaviour observation and a questionnaire. In the observation part, the user was asked to book two different types of travel products. The questionnaire contained 12 questions related to this booking task. The main results of this study are: a) the nature of the product searched influences the role of the product reviews, and b) advanced users, i.e., users who are familiar with online booking platforms and with consumeropinion platforms, are more interested in reviews expressing peculiar information such as a low (negative) rating about the product.

In the next chapter, chapter 2, we will discuss our research questions together with the applied research methodology. In chapter 3 we will present the test group composition, the decision models derived from the user study and the survey results. Chapter 4 will discuss the results and present the derived principles and finally, in chapter 5, we will present the conclusions and highlight future research work.

\section{Research Questions and Methodology}

The goal of this user behaviour study is to investigate the potential roles of product reviews (PRs) in the product selection process. The products considered in this study are hotels and activities. Our research ultimately aims at deriving good interaction guidelines for the designing of a new recommendation component based on product reviews. The research questions that we addressed are the following: 
1. In which part of the product selection process are PRs taken into account?

2. In what way do PRs complement other decision aid tools, like sorting, ranking and searching products?

3. Is it possible to generate new content, such as new product features, with reviews?

4. Are potential buyers more interested in positive or negative reviews?

5. When (subjective) reviews are regarded as an (objective) source of information? Does this depend of the amount of reviews, the average rating of the reviews, or the identification of the reader with the writer (shared views)?

We have articulated these questions into four hypotheses to test in our research:

- Hypothesis I: "The role of a product review depends on the stage of the decision process." The intuition behind this is that we believe that in the early stage of the decision process users are more focused on the product features and, as the decision process progresses, users will be more concerned with product reviews.

- Hypothesis II: "Negative reviews are of a higher importance compared to positive ones." This hypothesis is stating that a user is more interested in negative than positive product reviews, because the negative ones could reveal (potential) pitfalls.

- Hypothesis III: "The role of the reviews depends on the type of tourist product involved in the decision process." In fact, for more experience oriented products, like activities, and therefore harder to model with product features, reviews could be exploited even in the initial decision phase. Conversely, for products which could be modelled with a rich set of features, reviews could be better exploited in a later stage of the decision process, i.e., after that the user has reduced the options using the product features.

- Hypothesis IV: "The usage and impact of reviews depend on user characteristics". This hypothesis can be subdivided into four sub-hypothesis according to the tourist market segmentation literature, proposed by (Dibb et al., 1994) and (Swarbrooke \& Horner, 1999). The four components are: demographic characteristics, psychographic, socioeconomic and behaviourist.

The user behaviour study consisted of two parts. The first part is a behaviour observation study where the user is asked to book/select (fictitiously) two tourist products: a hotel and an activity. The location of the hotel and activity were supposed to be in the city centre of Amsterdam, and the user is supposed to stay in the hotel (fictitiously) for 3 nights and select one activity to visit. The total budget available 
was $€ 100$ per night for the hotel and $€ 40$ for the activity. We provided the user two different websites: Expedia and Tripadvisor. Product reviews are the main information content provided by Tripadvisor. Here the user can browse lists of products, grouped by the geographic location and product type and read other user opinions concerning these products. Only basic product features are displayed, such as price and location and it's not possible to query the product catalogue using the features of the product (e.g. "show the four stars hotels"). This limits the user's abilities to interact with this type of website. The second website involved in the test was Expedia. In Expedia, every hotel and activity is described by a rich set of wellknown features such as: number of rooms, price, and location. This structured product representation enables the user to issue queries and sort the available products according to her preferences. In this behaviour observation study we asked users to clearly write down the steps taken to accomplish the product selection task.

The second part of the study consisted of a questionnaire. The first goal was to get a global idea about the possible roles of reviews. The second goal was to check the consistency between the users' replies to the survey and the way they acted in the behaviour observation study. When we found some inconsistency we interpreted the data (of that specific user) as irrelevant. The questionnaire contained 12 questions with replies on a Likert scale ranging from 1 to 5 . The users involved in this study were students of the Tourism and Economics Master program of the Bocconi University. The education of the students is at least at the Bachelor's degree.

\section{$3 \quad$ Results}

In total 29 persons completed the survey but for data normalization issues we had to remove one entry and we analyzed 28 samples. General description of the sample is as following:

- Gender: 12 male and 16 female

- $\quad$ Age range: 22-37

- 23 Full time Master students and 5 Part time Master students.

- 8 persons used Expedia.com and/or Tripadvisor before

Next, we will first discuss the results of the hotel booking process and then the results of the activity booking task. In paragraph 3.3 we will present the results of the questionnaire.

\subsection{Hotel Decision Process}

In this section we present the hotel decision model, derived from the hotel booking observation study. The model is depicted in figure 1 . Note that we discarded 8 
samples of users who were not clear or ambiguous in their task description. Generally speaking, we can classify this booking process behaviour into 3 succeeding stages, according (Assael, 1984): information searching, information processing, alternative evaluation and purchase stage.

Information search stage. When we analyzed the results we saw for every user $(\mathrm{N}=21)$ a similar search pattern: start with Expedia and query for hotels in Amsterdam. This pattern shows that the users prefer to query the system with constraints (features) rather than browsing a list of (all the) hotels in Amsterdam, i.e., the Tripadvisor approach.

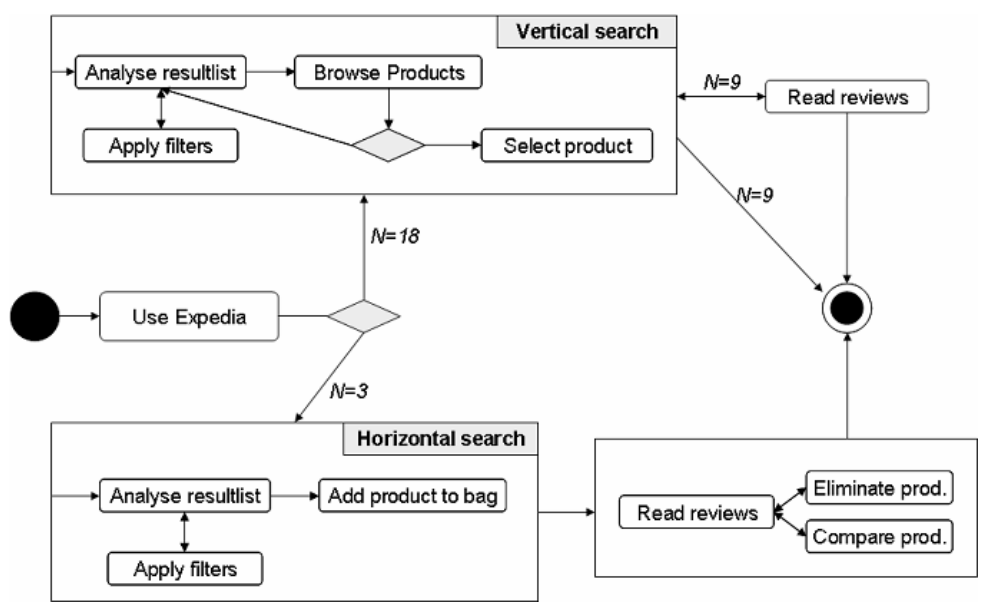

Fig. 1. Hotel booking decision model

Information processing- and evaluation stage. The initialization phase returns a list of hotels not constrained by the price. In fact, Expedia does not let the user to specify this constraint in the query. Therefore, to select those with a price smaller than $€ 100$, the subjects had to manipulate the result list. We noticed different approaches:

- Vertical search $(\mathbf{N}=\mathbf{1 8})$ In this approach, the users browsed the result list in an extensive way to find the best matching hotel. Best matching here means a hotel which satisfies the given budget constraints and offers the desired facilities. 9 Users incorporated Tripadvisor in there decision process to evaluate their choice. We noticed two roles: product confidence and product understanding. The first role was exploited when the user was positive about the initial hotel and wanted to know if there were any pitfalls regarding this hotel and the second role 
(product understanding) was exploited by users who did not have a specific hotel in mind, but wanted to know more about the recommended hotels.

- Horizontal search $(\mathbf{N}=3)$ In contrast to the vertical search approach in which product features are considered 'enough' to filter, we noticed users selecting a set of hotels which satisfied their basic needs, hereby stressing less on the specific product features. The final hotel selection was done with help of Tripadvisor. In this case, the role of Expedia is to provide an initial product filter. The (only) role of Tripadvisor we noticed is to filter the result set. We saw that the users based their decision both on the rating itself and the motivation of this review writer. The role of negative and positive reviews was only mentioned in one user task description. This user stated that she only looked at really positive and negative reviews

Purchase stage. In total, 12 user incorporated Tripadvisor, i.e. product reviews, in their decision process. 9 users did not consult Tripadvisor and based their decision only on the hotel features presented by Expedia. Finally, all the user succeeded in finding a suitable hotel according the given constraints

\subsection{Activity Decision Process}

In this section we present the activity decision model, derived from the activity booking observation study. The model is depicted in figure 2 . Note that we had to discard 6 samples of users who were not clear or ambiguous in their task description.

Information search stage. When analyzing the user search strategy, we noticed an interesting difference with respect to the hotel booking task: there were 6 users who consulted Tripadvisor directly without consulting Expedia before. The remaining, 16 users, still started with Expedia.

\section{Information processing- and evaluation stage}

- Users consulting Tripadvisor first $(\mathbf{N}=\mathbf{6})$. These users started the search process with Tripadvisor. 2 Users wrote explicitly down that they wanted to visit certain famous activities. These are users with background knowledge (Brucks, 1985), and they have already decided to visit a specific activity or a small set of activities. Major motivators mentioned for people to visit these activities are "status", in case the activity is internationally recognized, and a learning desire, in case it's a cultural activity (Swarbrooke \& Horner, 1999). In this case, the role of reviews is a better product understanding, since the tourist has already made a selection decision. The remaining users $(\mathrm{N}=4)$ who visited Tripadvisor directly 
without consulting Expedia, did not state they were looking for an activity they had in mind. None of these users consulted Expedia afterwards.

- Users consulting Expedia first ( $\mathbf{N}=\mathbf{1 6})$. The remaining group of 16 users, based their decision mainly on Expedia. This booking behaviour showed similarities with the hotel booking task: people searched on Expedia for activities in the neighbourhood of the hotel and got from Expedia an overview of the available activities. A drawback of Expedia is that it only offers popular choices, which are suited for the mass public but not always for the individual. But since the given task involves the booking of a short holiday, we think people were more biased to visit first the international, and therefore popular, famous activities as explained before. 11 people only consulted Expedia and based their decision on the information given by this site. 5 users consulted Tripadvisor after Expedia to verify their initial choice (product confidence).

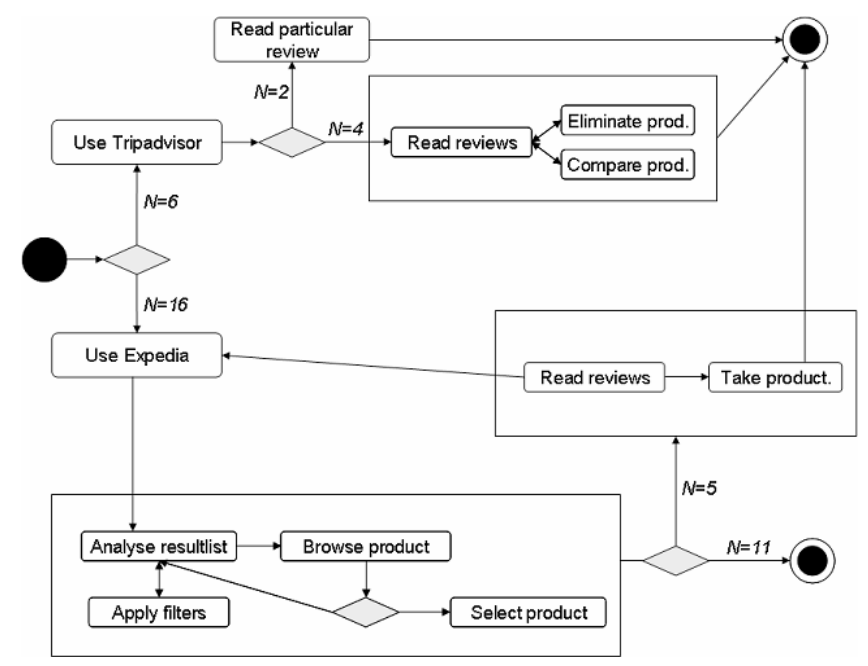

Fig. 2. Activity booking decision model

\subsection{Results of the Questionnaire}

In table 1 , the general results of the survey are presented. In total, the survey consisted of 12 questions with possible reply on a Likert scale ranging from 1 to 5 , where 1 represents totally disagree and 5 totally agree. We regard 3 as a neutral value.

To identify any difference in the replies between samples we have used t-test at $95 \%$ confidence level. In total, we have executed 3 tests, according to hypothesis IV. In the first test we checked any difference between male and female samples. The second was meant to identify any difference between the age groups and the last test we 
checked any difference between people who have never used an online booking systems vs. people who have used Expedia or Tripadvisor before.

Table 1. Questionnaire on product reviews usage

\begin{tabular}{|l|l|}
\hline Question & Average \\
\hline Q1: Reviews had an additional value in decision process & 3.11 \\
\hline Q2: I can book a hotel by only using reviews & 2.61 \\
\hline Q3: Decision is more based on reviews than information from travel guides & 3.21 \\
\hline Q4: Review rating is more important than motivation & 3.18 \\
\hline Q5: I am interested in highly rated reviews & 3.43 \\
\hline Q6: I would like to have a small set of reviews according my profile & 3.57 \\
\hline Q7: First select hotel, then show me the review & 3.68 \\
\hline Q8: I will use an recommender application with reviews on a mobile device & 2.39 \\
\hline Q9: I want to see all the reviews & 3.50 \\
\hline Q10: I am interested in negative reviews & 2.96 \\
\hline Q11: I first want to read a reviews and then select a hotel & 2.61 \\
\hline Q12: I will read review when I can't experience the product in the shop & 2.82 \\
\hline
\end{tabular}

Regarding the gender characteristic, we have found a significant difference in the replies to Q11 and Q12 in the two gender groups (male/female). Q11 is stating that a user first wants to read reviews and afterwards select a hotel. Women tend to disagree with an average reply of 2.06. Men, on the other hand, tend to agree and the average reply is 3.33. Q12 states that a user will only look at reviews in case the product is not available in the shop. Woman answered this question nearly unbiased (avg: 3.13). Men answered this question with an average of 2.33, stating that they will consult a review even if they can experience the product in a shop. One explanation could be that women are likely to have higher functional needs than men, (Vogt \& Fesenmaier, 1998) which are more likely to be found in the actual product description rather than in the product reviews

Regarding the experience factor, we have noticed a significant impact on the replies for Q9, Q10 and Q12. Inexperienced users tend to answer Q9 fairly positive (avg: 3.82), stating that they would like to see all the reviews compared to more experienced users who answer unbiased (avg: 3.00). Q10, is related to the user's interest in negative reviews, and were answered positively (avg: 3.64) by the experienced users. Inexperienced users tend to answer this question more negatively (avg: 2.53). The last significance difference was detected for Q12. This is not surprising since this question refers to the role of reviews in case the product can be experienced in a shop. Experienced users answered that they will still read the review (avg: 2.27) whereas the inexperienced users were nearly unbiased. General speaking, experienced users seem to have more ideas about product reviews than inexperienced 
users. One explanation for this could be that inexperienced user rely more on professional (expert) sources than experienced ones. (Snepenger et al., 1990; Woodside \& Ronkainen, 1980)

\section{Summary and Discussion}

As we noticed in the task analysis presented in the previous section, there are different roles played by product reviews in the decision process. These roles vary according to the circumstances of each product selection process. This implies that both Hypothesis 1 and 3 are confirmed. In the hotel booking task, the reviews were consulted at the end of the decision process, mainly for increasing confidence. Moreover, if the user is not biased towards a product the reviews can increase product understanding. We noticed this behaviour when the user selected initially more products. In contrast with the hotel booking process, in the activity selection task we noticed that the reviews were invoked even at the beginning of the decision process. We think this is due to the difference in the product characteristics. Activities have a larger experiential nature and are harder to model with objective features. Hotels on the other hand are more like services, and they can described with some service characteristics, like the availability of a swimming pool, size of the rooms etc. One aspect which is shared by both decisions processes, and that can be generalized, is the need for some basic product features like location and type of product. When we compare these results with those of the user survey, we notice that most people first want to see the product description and then would read the reviews. This yields that the role of the reviews depends on the stage of the decision process, but also on the type of tourist product involved. This means that we can both accept Hypothesis I and III.

Concerning our initial hypothesis II, stating that negative reviews are more important than positive ones in the decision process, we have to reject this hypothesis since we have not found any significant difference. We would like to say that the type of the review has an impact on the role it can play, e.g., positive reviews are more suitable for product understanding and for increasing the product knowledge whereas negative ones are more useful for product decision confidence.

Regarding hypothesis IV, we can confirm that the usage and impact of reviews depend on the user characteristics. In fact, we saw a significant difference between the way men and women interact with the reviews. Men are more inclined to trust the opinion expressed in a review, whereas women seem less influenced by reviews and base their decision more on their own product interpretation. Conversely, we have not found significant differences determined by the age. Considering the last variable experience - we saw some interesting differences: users without the application 
experience wanted to see all the reviews instead of a best-fitting selection. Another interesting aspect is that experienced users were highly interested in negative reviews, which could be one of the motivations to visit sites like Tripadvisor. The conclusion is that these, although basic, user characteristics already show that there is a difference in how people process reviews and therefore, we should take these differences in account when offering reviews.

The next design principles are derived from the results of the user behaviour study and the questionnaire. These principles will be used in the recommender system we are building:

- User should be able to change the way reviews are presented, for instance use a personalized filtering approach or show all the reviews.

- The system has to offer functionalities to manipulate the product review list, which means: to show the most positive or negative reviews.

- The system should offer the possibility to enter initial constraints or using the user's profile to generate the first product recommendations.

- In the product recommendation (overview) the user should be able to read the product reviews for those products she is considering.

- The user should be able to browse efficiently the different product reviews.

- The user should be able to consult the reviews directly (inspiring mode) without retrieving the products first.

- The user should be able to browse products and related product reviews in an efficient way (few interaction steps). This aspect supports the vertical search approach.

- User should be able to add products to a product bag and filter the reviews according to this product bag. (horizontal search approach).

\section{Conclusions and Future Work}

In this paper we have presented the results of a user behaviour study where we investigated the possible roles of product reviews in travel decision making and in recommender systems. We argued that product reviews can give a better product understanding, increase trust and the feeling of having an objective evaluation. These are all important aspects for the tourist domain due to the specific characteristics of the products (service oriented). The main results of our study so far is that we found a difference in the role of reviews related to the product involved and we discovered that advanced users, i.e. those who are familiar with online booking platforms and with consumer-opinion platforms, are more interested in reviews with a low 
(negative) rating. In the future, we will use the design guidelines of this study for a new recommender methodology for mobile systems.

\section{References}

Assael, H. (1984). Consumer behaviour and marketing action. Boston: Kent.

BBC (2004). Net shopping guides lure surfers, url: http://news.bbc.co.uk/1/hi/technology/3736668.stm

Blackshaw, P. (2005). The Pocket Guide to Consumer Generated Media. Clikz Experts. Url: http://www.clickz.com/experts/brand/cmo/article.php/3515576

Blackshaw, P., and Nazzaro, M. (2005). Consumer-generated media 101: Word-of-mouth in the age of the web-fortified consumer. White paper, Intelliseek

Brucks, M. (1985). The effects of product class knowledge on information search behavior. Journal of Consumer Research, 12: 1-16.

Buhalis, D. (2003). eTourism: Information Technology for Strategic Tourism Management. Prentice Hall.

Dibb, S., Simkin, L., Pride, W.M., and Ferrel, O.C. (1994). Marketing concepts and Strategies. Houghton-Mifflin, London, UK.

Hennig-Thurau, T., Gwinner, K., Walsh, G., and Gremler,D. (2004) Electronic word-of-mouth via consumer-opinion platforms. Journal of Interactive Marketing, Vol.18, Nr.1, 2004.

Jin, Y., Bloch, B., and Cameron, G (2002). A comparative study: Does the word-of-mouth communications and opinion leadership model fit epinions on the internet? Proceedings of the Hawaii International Conference on Social Sciences.

PR Newswire Europe Ltd (2005). How to 'Wynn' in Las Vegas http://www.prnewswire.co.uk/cgi/news/release?id=145574

Ricci, F. (2002). Travel recommender systems. IEEE Intelligent Systems, 17(6):55-57.

Saranow J. (2004). Getting Travel Advice From a Stranger. The Wall Street Journal, Sep 28.

Schafer, J. B., Konstan, J. A., and Riedl, J. (2001). E-commerce recommendation applications. Data Mining and Knowledge Discovery, 5(1/2):115-153.

Senecal S., Nantal A. (2004). The influence of online product recommendations on consumers' online choices. Journal of Retailing, $\mathrm{Nr}$ 80: 159-169.

Snepenger, D., K. Meged, M. Snelling, and K. Worral (1990). Information search strategies by destination-naïve tourists. Journal of Travel Research, 29: 13-16.

Swarbrooke J., and Horner S. (1999). Consumer Behavior in Tourism. Butterworth and Heinemann.

Tripadvisor, http://www.tripadvisor.com

Vogt, C. A., and D. R. Fesenmaier (1998) Expanding the functional information search model. Annals of Tourism Research, 25(3): 551-578.

Woodside, A. G., and I. A. Ronkainen (1980). Vacation planning segments: Self-planning vs. users of Motor Club and travel agents, Annals of Tourism Research, 7: 385-393.

\section{Acknowledgments}

We would like to thank Prof. Rodolfo Baggio and his 29 Master of Tourism and Economics students for having participated in this user behaviour study. René Wietsma's research work was funded with a Leonardo (EU) placement, contract number: "NL/04/A/PL/HO/123040". 